\title{
Determine Image Quality Enhanced by Using the Suggested Algorithm
}

\author{
Firas S. Abdulameer \\ Department of Physics, Collage of Sciences, Mustansiriyah University, PO box 46007, Iraq-Baghdad
}

\begin{abstract}
In this research has been improved the brightness and contrast through a new algorithm suggestion based on the algorithm retinex and algorithm settle histogram. Use retinex algorithm for homogeneous regions and algorithm settle histogram for edge regions and using effective Sobel. The comparison showed between the three methods by calculating the quality standards $(\mu, \sigma)$ and by observing the results that the Suggested method has successfully in enhancement brightness and contrast are almost equal compared with other methods.
\end{abstract}

Keywords: Image quality, Scale retinex, Histogram, Mean, Standard deviation, Brightness, Contrast.

DOI: $10.7176 /$ APTA/76-04

Publication date:March $31^{\text {st }} 2019$

\section{Introduction}

The image quality of the indicates its degree of clarity, and can be expressed by image analysis or analysis ability, which can be defined as the smallest angle separation between two bodies so that remain separate from each other in the visual system, Image quality can be defined as the quantity of sharpness and contrast in image details [1]. Where considered contrast is a factor affecting the image quality, known as the dynamic range of the display system, is simply a way of expressing the difference degree between the brightest gradients most and subtle in the picture [2], where the contrast is two types: local contrast the contrast of each small part of the image, and the global contrast is the ratio spatial contrast small parts of the image, the picture with high general contrast means that the picture contains high detail and rich in changes .in return for, the picture with little general contrast has fewer details and has gradual variations difficult to detect visually $[3,4]$.

\section{Evaluation image quality by a scale $(\mu, \sigma)$}

Evaluation of image quality Based on the mean and standard deviation, we divided the image into several blocks $15^{*} 15$ and size 50 pixels, $\mathrm{i}$ Represents the number of partition regions, we taken the mean of $\mu \mathrm{i} \sigma \mathrm{i}$ is for each block and then calculated, each $\bar{\mu} \bar{\sigma}$, Which represent the average both of $\mu \mathrm{i}$, $\sigma \mathrm{i}$ for all blocks(partition

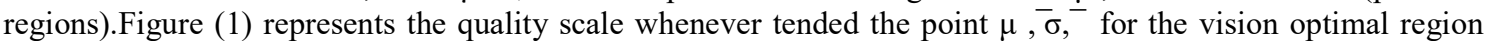
Quality has become the highest here is enhanced of lighting and contrast. When the point in the insufficient contrast region, there is an enhanced of the lighting only and vice versa in the insufficient lightness region where there is only enhanced in contrast [5].Quality has become the highest here is enhanced of lighting and contrast. When the point in the insufficient contrast region, there is an enhanced of the lighting only and vice versa in the insufficient lightness region where there is only enhanced in contrast [5].

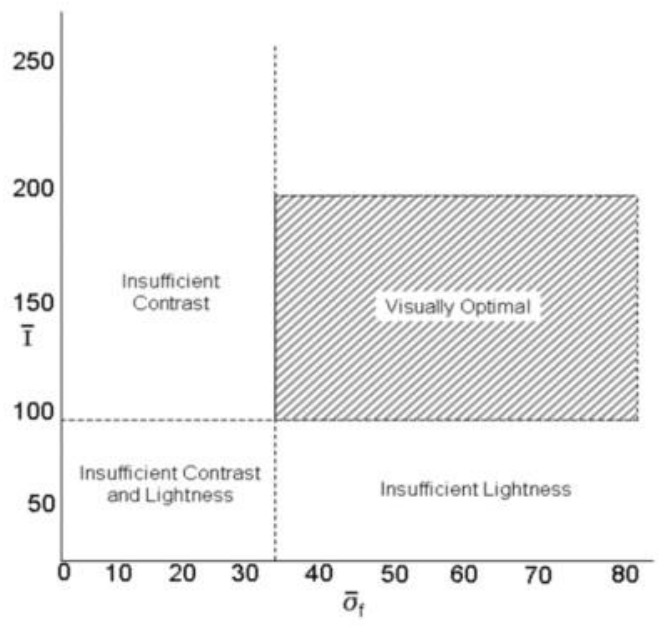

Figure1. Describe image quality between Mean and standard deviation $(\mu, \sigma)[5]$.

\section{Multi Scale Retinex Algorithm with Color Restoration MSRCR}


The multiscale retinex (MSR) is explained from single-scale retinex (SSR) we have [7]:

$$
R_{i}(x, y, c)=\log \left(I_{i}(x, y)\right]-\log \left[F(x, y, c) * I_{i}(x, y)\right]
$$

Where $R_{i}(x, y, c)$ the output of component $\mathrm{i}(\mathrm{i} \in \mathrm{R}, \mathrm{G}, \mathrm{B})$ at position $\mathrm{x}, \mathrm{y}, \mathrm{c}$ is the standard division of the Gaussian function , $I_{i}(x, y)$ is the image value for component $\mathrm{i}$ and symbol * denoted convolution .

$F(x, y, c)$ Gaussian function that is calculated by [7]:

$$
\mathrm{F}(\mathrm{x}, \mathrm{y}, \mathrm{c})=\operatorname{kexp}\left(\frac{-\left(\mathrm{x}^{2}-\mathrm{y}^{2}\right)}{\mathrm{c}^{2}}\right)
$$

The constant $\mathrm{k}$ is determined by:

$$
\iint F(x, y, c) d x d y=1
$$

The MSR output is then a weighted sum of the outputs of several different SSR output where [2]:

$$
R_{M S R}(x, y, w, c)=\sum_{n=1}^{N} W_{n} R_{i}\left(x, y, c_{n}\right)
$$

Where $\mathrm{N}$ is the number of scales, $R_{i}\left(x, y, c_{n}\right)$ the i'th component of the n'th scale, $R_{M S R}(x, y, w, c)$ the i'th spectral component of the MSR output and $W_{n}$. We insist that $\sum W_{n}=1$. The result of the above processing will have both negative and positive RGB values, and the histogram will be typically have large tails. Thus a final gain-offset has been applied as mentioned in [7]. This processing can be cause image colors to go towards gray, and thus an additional processing step is proposed in [2]:

Where $\widehat{I}_{l}$ given by [2]:

$$
R^{\prime}=R_{M S R} I_{i}(x, y)
$$

$$
\widehat{I}_{l}(x, y, a, b)=b \log \left[1+a \frac{I_{i}(x, y)}{\sum_{i=1}^{3} I_{i}(x, y)}\right.
$$

Where we have taken the liberty to use $\log (1+x)$ in place of $\log (x)$ due to a positive result. In [2] a value of 125 is suggested for (a ); we empirically settled on a value of $(b=100)$ for a specific test image. The difference between using these two values is small. In equation (7) a second constant is used which is simply a multiplier of the result. And the final step is gain-offset by 0.35 and 0.56 respectively. The present research uses: $(\mathrm{w} 1=\mathrm{w} 2=\mathrm{w} 3=1 / 3)$ and $(\mathrm{c} 1=250, \mathrm{c} 2=120, \mathrm{c} 3=80)[2]$.

\section{Modified Retinex Algorithm MR}

In the MR the color image is transformed from basic RGB color space to YIQ color space, the forward transform is given by [10]:

$$
\left.\begin{array}{l}
Y=0.298 R+0.587 G+0.114 B \\
I=0.596 R-0.270 G+0.233 B \\
Q=0.221 R+0.253 G+0.312 B
\end{array}\right\}
$$

Where y is lightness, component, i,q are chromatic components. In second step is transform the normalized lightness value by using sigmoid function hat is given by[3]:

$$
Y_{t}=1 /\left(1+\sqrt{\frac{1-Y n}{Y n}}\right)
$$

Where Yn being the normalized lightness value that is equal (Y/255). Then used the inverse transformation from YIQ to RGB color space calculated in $Y p I Q$ that is given by [10]:

$$
\begin{aligned}
& r_{p}=y_{p}+0.956 i+0.621 q \\
& g_{p}=y_{p}-0.272 i-0.647 q \\
& b_{p}=y_{p}-1.106 i+1.703 q
\end{aligned}
$$

And the MSRCR had been applied on eq. (10)[3].

\section{Suggested algorithm MRHE}

In this study, a new algorithm suggestion to enhance the illumination and contrast in the color image by Merge algorithms retinex and the histogram equalization (MRHE), first applied retinex Algorithm According to the relations above-mentioned, then Detection of edges using the effect sobel within:

So the can be selected edge of the image using:

$$
\begin{aligned}
G_{x} & =\left[\begin{array}{ccc}
-1 & 0 & -1 \\
-2 & 0 & -2 \\
-1 & 0 & -1
\end{array}\right] \\
G_{y} & =\left[\begin{array}{ccc}
1 & 2 & 1 \\
0 & 0 & 0 \\
-1 & -2 & -1
\end{array}\right]
\end{aligned}
$$

$$
I_{x}=I \otimes G_{x}
$$




$$
\begin{gathered}
I_{y}=I \otimes G_{y} \\
I e=\max \left(I_{x}, I_{y}\right)
\end{gathered}
$$

Then enhance the values of the edges only by applying histogram equalization algorithms.

The suggestion method as shown in Figure (2)

$$
(15) I_{e}(x, y)= \begin{cases}I_{R}(x, y) & \text { non edge } \\ I_{h}(x, y) & \text { if edge }\end{cases}
$$

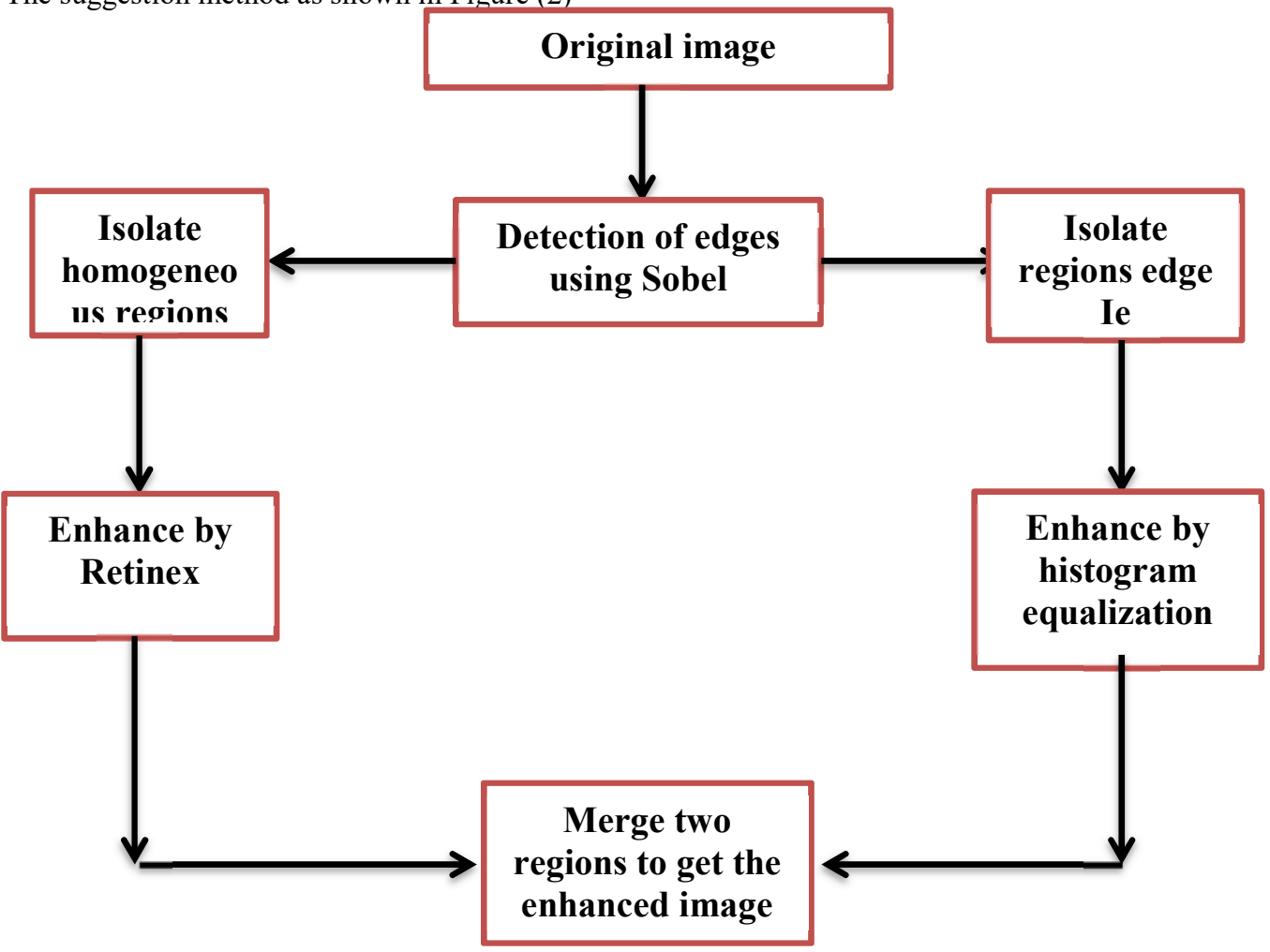

Figure2. Suggested algorithm diagram

\section{Results and discussion}

In this research, were enhanced the color images and used three images a size $(540 \times 720)$ of type JPG [10]. As shown in figure (3), and used Matelab Ra2013 in those images. Figure (4) shows images original and enhanced using methods (HE,MSRCR,MRHE) According to the observation, we find that the best method is the proposed method where was an improvement in light and contrast reflect in the calculation of quality because preserve the contrast in the edge regions and enhance the lighting in homogeneous regions, in figure (5) represents the evaluation of the quality image enhanced by criteria $(\mu, \sigma)$. Note that the improvement points went to the efficiency region to improve the lighting and contrast, at last the proposed algorithm (MRHE) is the best followed by the Retinex algorithm and the histogram (HE).

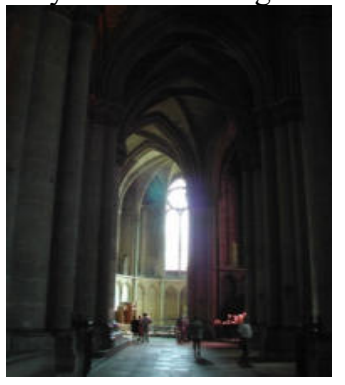

$\mathrm{a}$

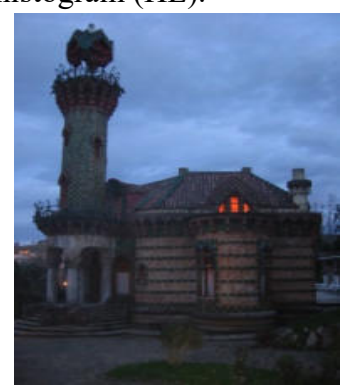

b

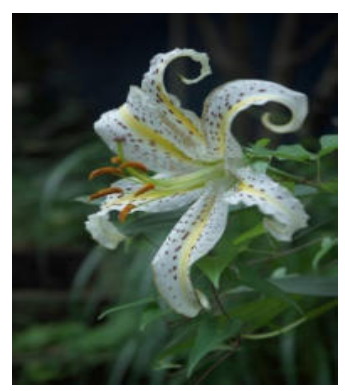

$\mathrm{c}$

Figure3. Original color images (a,b,c) [10]. 


\section{Conclusions}

Through the results the proposed method (MRHE) succeeded in enhancing the lightness and contrast in images by using the scale $(\mu, \sigma)$ which is better in enhancing compared to other methods Retinex (MSRCR) which are lower and then followed by the histogram (HE), which enhanced the homogeneous region, as well as enhanced lighting and contrast in parallel.

\section{References}

[1] John.R .Jenson (1986). Introductory Digitial Image Processing A Remote Sensing Perspective, Prentice Hall , Engle Wood Clifs, New -Jersey 07632.

[2] Barten D.G.J., (1999). Contrast Sensitivity OF Human Eye and its Effect on Image Quality, SPIE - The International Society For Optical Engineering Belling Ham.

[3] NeumannL., Matkovick, Purgathafer W. (2002). The Global Contrast Factor , in proceeding of the first seminar on Computer Graphics and Visualization, P.P 108 - 119 .

[4] Neumann L., Sbert M., Gooch B., (2005). Global Contrast factor - Anew approach to image contrast ,Computational Aesthetics in Graphics, Visualization and Imaging,.

[5] D.Jabson, Z.Rahman,G.A Woodell,(2002).Statistics of visual representation,Proc.SPIE4736, pp25- 35.

[6] D.Jabson, Z.Rahman, and G.A. Woodel,( July 1997).A multi-scale retinex for bridging the gapbetween color images and the human observation of scenes," IEEE Trans. Image Process. 6,pp. 965-976.

[7] D. J. Jobson, Z. Rahman, and G. A. Woodell, (1996). Properties and performance of a center/surround retinex, IEEE Trans. on Image Processing 6, pp. 451-462, March

[8] Sony Wine S., J and Horne R.E., (1998).The Color Image Processing Hand Book, International Thomson.

[9] Ali A . Al - Zuky ,Hazim G Daway, Hana H. Kareem , Firas S Mohammed (2010) Aerial image enhancement using modified retinex algorithm, IEEE International Conference on Advanced Computing \& Communication Technologies (ICACCT).

[10] http://demo.ipol.im/demo/131/. 


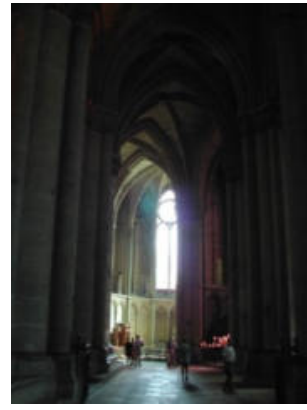

(a) Orginal

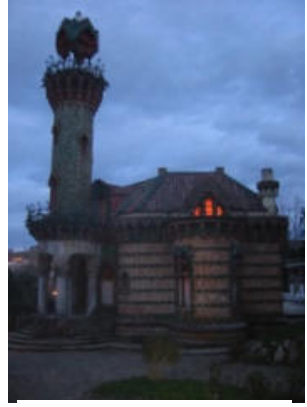

(b) Orginal

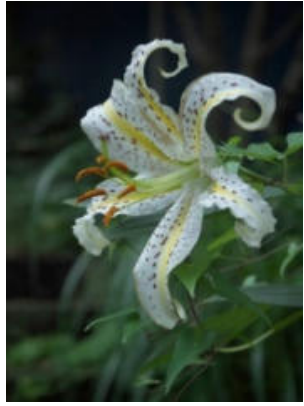

(c) Orginal

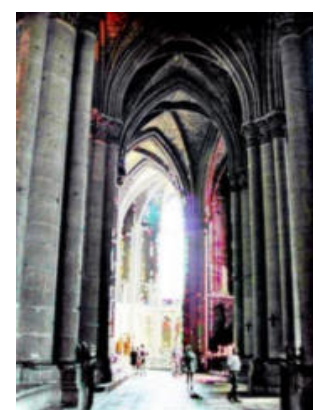

(a1) $\mathrm{HE}$

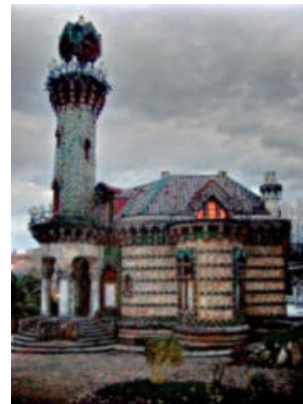

(b1) HE

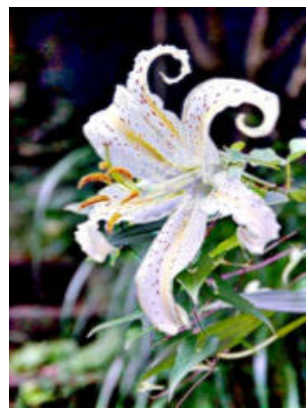

(c1) HE

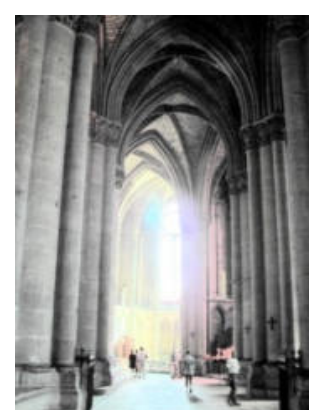

(a2)MSRCR

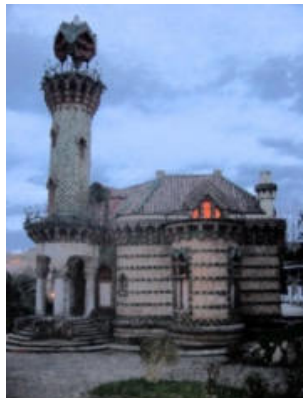

(b2)MSRCR

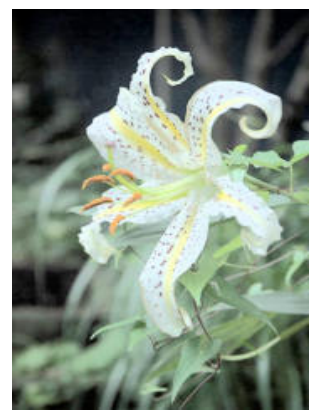

(c2)MSRCR

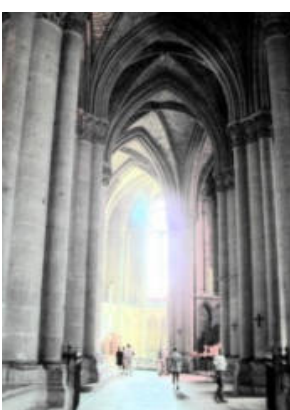

(a3)MRHE

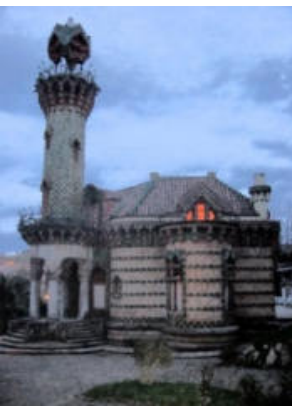

(b3)MRHE

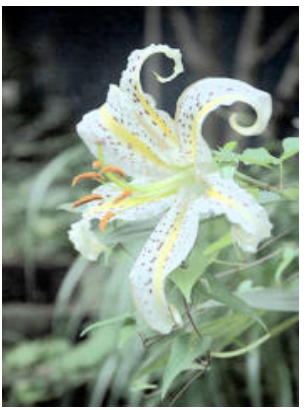

(c3)MRHE

Figure4. The original images and enhanced and result from the three methods is the histogram (HE), retinex (MSRCR), proposed (MRHE) 


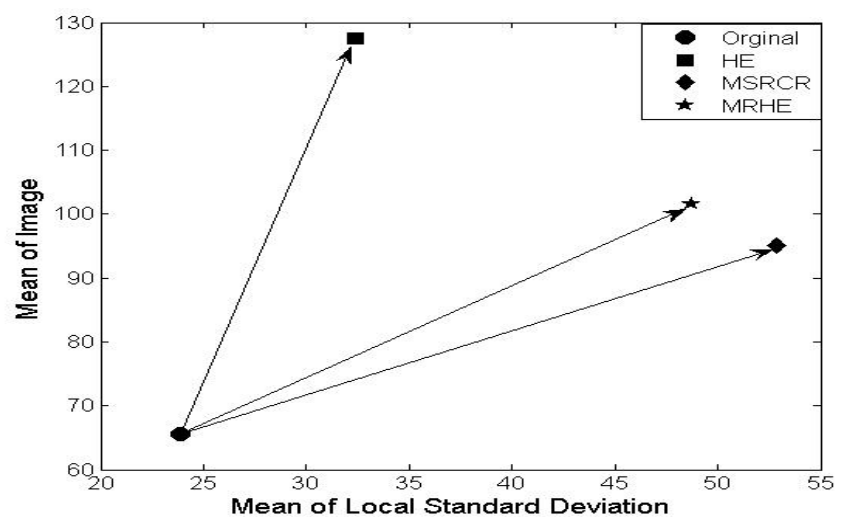

Figure5a. The original image (a) and enhanced by three methods (a1, a2, a3)

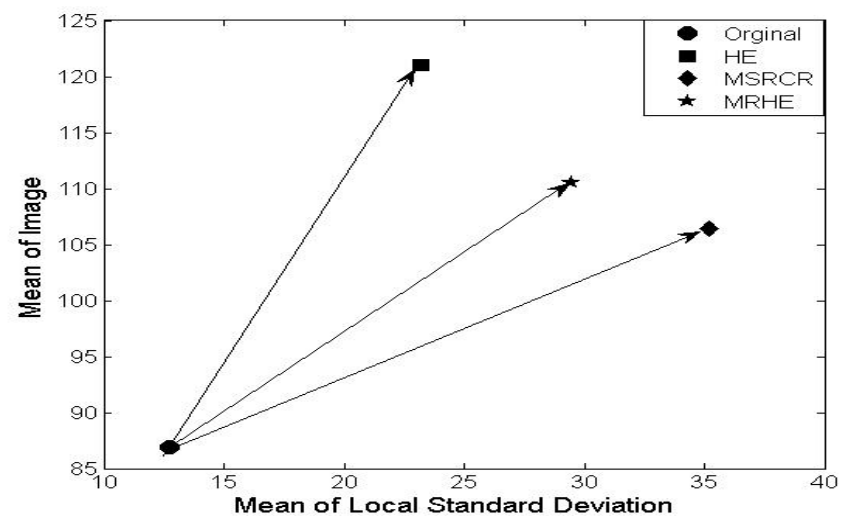

Figure 5b. The original image (b) and enhanced by three methods (b1, b2, b3)

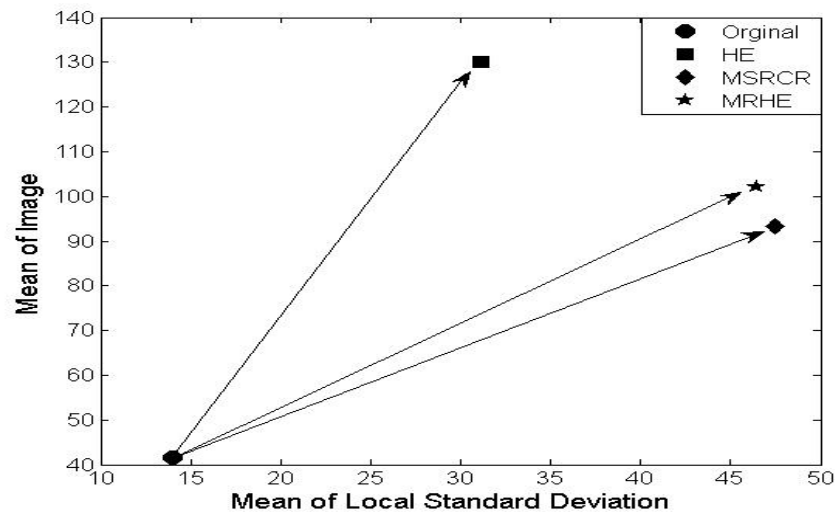

Figure5c. The original image (c) and enhanced by three methods (c1, c2, c3)

Figure5. Results of evaluate the enhanced images quality through the relationship between Mean and Standard deviation $(\mu, \sigma)$. 